\title{
Some Fixed Point Theorems in $b$-Metric Space Endowed with Graph
}

\author{
Maria Samreen, ${ }^{1}$ Tayyab Kamran, ${ }^{2}$ and Naseer Shahzad ${ }^{3}$ \\ ${ }^{1}$ Centre for Advanced Mathematics and Physics, National University of Sciences and Technology, Sector, H-12, Islamabad, Pakistan \\ ${ }^{2}$ Department of Mathematics, Quaid-i-Azam University, Islamabad, Pakistan \\ ${ }^{3}$ Department of Mathematics, King Abdulaziz University, Jeddah, Saudi Arabia
}

Correspondence should be addressed to Naseer Shahzad; nshahzad@kau.edu.sa

Received 6 July 2013; Revised 5 September 2013; Accepted 6 September 2013

Academic Editor: Douglas Anderson

Copyright (C) 2013 Maria Samreen et al. This is an open access article distributed under the Creative Commons Attribution License, which permits unrestricted use, distribution, and reproduction in any medium, provided the original work is properly cited.

We define some notions of contraction mappings in $b$-metric space endowed with a graph $G$ and subsequently establish some fixed point results for such classes of contractions. According to the applications of our results, we obtain fixed point theorems for cyclic operators and an existence theorem for the solution of an integral equation.

\section{Introduction and Preliminaries}

The study of $b$-metric spaces was initiated in some works of Bakhtin, Heinonen, Bourbaki, and Czerwik [1-4]. Afterwards, several articles which deal with fixed point theorems for single-valued and multivalued functions in $b$-metric space appeared [1-8].

Definition 1 (see $[1,4]$ ). Let $X$ be a set, and let $s \geq 1$ be a given real number. A function $d: X \times X \rightarrow \mathbb{R}^{+}$is said to be a $b$-metric on $X$, and the pair $(X, d)$ is called a $b$-metric space if, for all $x, y, z \in X$,

(d1) $d(x, y)=0$ if and only if $x=y$,

(d2) $d(x, y)=d(y, x)$,

(d3) $d(x, z) \leq s[d(x, y)+d(y, z)]$.

Note that the class of $b$-metric spaces contains the class of metric spaces.

Example 2. Let $X:=l_{p}(\mathbb{R})$ with $0<p<1$, where $l_{p}(\mathbb{R}):=$ $\left\{x=\left\{x_{n}\right\} \subset \mathbb{R}: \sum_{n=1}^{\infty}\left|x_{n}\right|^{p}<\infty\right\}$. Then, $d(x, y)=\left(\sum_{n=1}^{\infty} \mid x_{n}-\right.$ $\left.\left.y_{n}\right|^{p}\right)^{1 / p}$ is a $b$-metric on $X$ with $s=2^{1 / p}$.
A sequence $\left\{x_{n}\right\}$ in a $b$-metric space $X$ is said to be convergent if and only if there exists $x \in X$ such that $d\left(x_{n}, x\right) \rightarrow 0$ as $n \rightarrow \infty$. In this case, we write $\lim _{n \rightarrow \infty} x_{n}=$ $x$. A sequence $\left\{x_{n}\right\}$ in a $b$-metric space $X$ is said to be Cauchy if and only if $d\left(x_{n}, x_{m}\right) \rightarrow 0$ as $m, n \rightarrow \infty$. A $b$-metric space $(X, d)$ is complete if every Cauchy sequence in $X$ converges. In general, a $b$-metric is not continuous.

The famous Banach contraction principle [9] infers that every contraction on a complete metric space has a unique fixed point. Recently, Jachymski [10] introduced the notion of Banach $G$-contraction to generalize Banach contraction principle as follows. Let $(M, \delta)$ be a metric space, let $\Delta$ be the diagonal of the Cartesian product $M \times M$, and let $G$ be a directed graph such that the set $V(G)$ of its vertices coincides with $M$ and the set $E(G)$ of its edges contains all loops; that is, $E(G) \supseteq \Delta$. Assume that $G$ has no parallel edges. A mapping $f: M \rightarrow M$ is called a Banach $G$-contraction if (i) for all $x, y \in X((x, y) \in E(G) \Rightarrow(f x, f y) \in E(G))$, (ii) $\exists \alpha$, $0<\alpha<1$ such that for all $x, y \in X,(x, y) \in E(G) \Rightarrow$ $\delta(f x, f y) \leq \alpha \delta(x, y)$. A mapping $f: M \rightarrow M$ is known as Picard operator [11] if $f$ has a unique fixed point $x^{*}$ and $\lim _{n \rightarrow \infty} f^{n} x=x^{*}$ for all $x \in M$.

Various generalizations of Banach's principle have been obtained by weakening contractive conditions. In this context, Matkowski [12] introduced class of $\varphi$-contractions in 
metric fixed point theory, and subsequently further study was developed in this setting by different authors when underlying space was taken to be a partially ordered set (see, e.g., $[13,14])$.

Let $\varphi: \mathbb{R}^{+} \rightarrow \mathbb{R}^{+}$. Consider the following properties:

(i) $t_{1} \leq t_{2} \Rightarrow \varphi\left(t_{1}\right) \leq \varphi\left(t_{2}\right)$, for all $t_{1}, t_{2} \in \mathbb{R}^{+}$,

(ii) $\varphi \varphi(t)<t$ for $t>0$,

(iii) $\varphi(0)=0$,

(iv) $\lim _{n \rightarrow \infty} \varphi^{n}(t)=0$ for all $t \geq 0$,

$(\mathrm{v})_{\varphi} \sum_{n=0}^{\infty} \varphi^{n}(t)$ converges for all $t>0$.

It is easily seen that (i) $)_{\varphi}$ and (iv) $)_{\varphi}$ imply $(\text { ii) })_{\varphi}$ and (i) $)_{\varphi}$ and (ii) $)_{\varphi}$ imply $(\mathrm{iii})_{\varphi}$.

We recall that a function $\varphi$ satisfying $(\mathrm{i})_{\varphi}$ and (iv) $)_{\varphi}$ is said to be a comparison function. A function $\varphi$ satisfying $(\mathrm{i})_{\varphi}$ and $(\mathrm{v})_{\varphi}$ is known as $(c)$-comparison function.

Any $(c)$-comparison function is a comparison function, but converse may not be true. For example, $\varphi(t)=t /(1+t)$; $t \in \mathbb{R}^{+}$is a comparison function but not a (c)-comparison function. On the other hand, define $\varphi(t)=t / 2 ; 0 \leq t \leq 1$ and $\varphi(t)=t-(1 / 2) ; t>1$, and then $\varphi$ is a $(c)$-comparison function. For details on $\varphi$ contractions, we refer the readers to $[15,16]$.

Berinde [17] took further step to investigate $\varphi$ contractions when the framework was taken to be a $b$-metric space, and for some technical reasons, he had to introduce the notion of $b$-comparison function; in particular, he obtained some estimations for rate of convergence [17]. For other related results, see also [5, 7, 17-21].

Definition 3. Let $s \geq 1$ be a fixed real number. A function $\varphi$ : $\mathbb{R}^{+} \rightarrow \mathbb{R}^{+}$is known as $b$-comparison function if it satisfies (i) ${ }_{\varphi}$, and the following holds:

$$
\text { (vi) } \sum_{\varphi=0}^{\infty} s^{n} \varphi^{n}(t) \text { converges for all } t \in \mathbb{R}^{+} \text {. }
$$

The concept of $b$-comparison function coincides with comparison function when $s=1$. Let $(X, d)$ be a $b$-metric space with coefficient $s \geq 1$, and then $\varphi(t)=a t ; t \in \mathbb{R}^{+}$with $0<a<(1 / s)$ is a $b$-comparison function.

\section{Main Results}

Throughout this section, let $(X, d)$ be a $b$-metric space with coefficient $s \geq 1$, and $\Delta$ is the diagonal of the Cartesian product $X \times X$. $G$ is a directed graph such that the set $V(G)$ of its vertices coincides with $X$, and the set $E(G)$ of its edges contains all loops; that is, $E(G) \supseteq \Delta$. Assume that $G$ has no parallel edges. We assign to each edge having vertices $x$ and $y$ a unique element $d(x, y)$. Now, we introduce the following definition.

Definition 4. One says that a mapping $f: X \rightarrow X$ is a $b$ $(\varphi, G)$ contraction if for all $x, y \in X$ :

$$
(f x, f y) \in E(G) \quad \text { whenever }(x, y) \in E(G)
$$

$$
d(f x, f y) \leq \varphi(d(x, y)) \quad \text { whenever }(x, y) \in E(G),
$$

where $\varphi: \mathbb{R}^{+} \rightarrow \mathbb{R}^{+}$is a comparison function.

Remark 5. Note that a Banach $G$-contraction is a $b-(\varphi, G)$ contraction.

Example 6. Any constant mapping $f: X \rightarrow X$ is a $b-(\varphi, G)$ contraction for any graph $G$ with $V(G)=X$.

Example 7. Any self-mapping $f$ on $X$ is trivially a $b$ - $\left(\varphi, G_{1}\right)$ contraction, where $G_{1}=(V(G), E(G))=(X, \Delta)$.

Example 8. Let $X=\mathbb{R}$, and define $d: X \times X \rightarrow \mathbb{R}$ by $d(x, y)=|x-y|^{2}$. Then, $d$ is a $b$-metric on $X$ with $s=2$. Further, $f x=x / 2$, for all $x \in X$. Then, $f$ is a $b-\left(\varphi, G_{0}\right)$ contraction with $\varphi(t)=t / 4$ and $G_{0}=(X, X \times X)$. Note that $d$ is not a metric on $X$.

Definition 9. Two sequences $\left\{x_{n}\right\}$ and $\left\{y_{n}\right\}$ in $X$ are said to be equivalent if $\lim _{n \rightarrow \infty} d\left(x_{n}, y_{n}\right)=0$, and if each of them is a Cauchy sequence, then they are called Cauchy equivalent. lemma.

As a consequence of Definition 9, we get the following

Remark 10. Let $\left\{x_{n}\right\}$ and $\left\{y_{n}\right\}$ be equivalent sequences in $X$. (i) If $\left\{x_{n}\right\}$ converges to $x$, then $\left\{y_{n}\right\}$ also converges to $x$ and vice versa. (ii) $\left\{y_{n}\right\}$ is a Cauchy sequence whenever $\left\{x_{n}\right\}$ is a Cauchy sequence and vice versa.

Now, we recollect some preliminaries from graph theory which we need for the sequel. Let $G=(V(G)$, and let $E(G))$ be a directed graph. By letter $\widetilde{G}$, we denote the undirected graph obtained from $G$ by ignoring the direction of edges. If $x$ and $y$ are vertices in a graph $G$, then a path in $G$ from $x$ to $y$ of length $l$ is a sequence $\left\{x_{i}\right\}_{i=0}^{l}$ of $l+1$ vertices such that $x_{0}=x, x_{l}=y$, and $\left(x_{i-1}, x_{i}\right) \in E(G)$ for $i=1, \ldots, l$. A graph $G$ is called connected if there is a path between any two vertices. $G$ is weakly connected if $\widetilde{G}$ is connected. For a graph $G$ such that $E(G)$ is symmetric and $x$ is a vertex in $G$, the subgraph $G_{x}$ consisting of all edges and vertices which are contained in some path beginning at $x$ is known as a component of $G$ containing $x$. So that $V\left(G_{x}\right)=[x]_{\widetilde{G}}$, where $[x]_{\widetilde{G}}$ is the equivalence class of a relation $R$ defined on $V(G)$ by the rule: $y R z$ if there is a path in $G$ from $y$ to $z$. Clearly, $G_{x}$ is connected. A graph $G$ is known as $(C)$-graph in $X$ [22] if for any sequence $\left\{x_{n}\right\}$ in $X$ with $x_{n} \rightarrow x$ and $\left(x_{n}, x_{n+1}\right) \in E(G)$ for $n \in \mathbb{N}$; then there exists a subsequence $\left\{x_{n_{k}}\right\}$ of $\left\{x_{n}\right\}$ such that $\left(x_{n_{k}}, x\right) \in E(G)$ for $k \in \mathbb{N}$.

Proposition 11. Let $f: X \rightarrow X$ be a $b-(\varphi, G)$ contraction, where $\varphi: \mathbb{R}^{+} \rightarrow \mathbb{R}^{+}$is a comparison function; then

(i) $f$ is $a b-(\varphi, \widetilde{G})$ contraction and $a b-\left(\varphi, G^{-1}\right)$ contraction as well,

(ii) $\left[x_{0}\right]_{\widetilde{G}}$ is $f$-invariant, and $\left.f\right|_{\left[x_{0}\right]_{\widetilde{G}}}$ is a $b-\left(\varphi, \widetilde{G}_{x}\right)$ contraction provided that $x_{0} \in X$ is such that $f x_{0} \in\left[x_{0}\right]_{\widetilde{G}}$. 
Proof. (i) By using (d2) (Definition 1) it can be easily proved.

(ii) Let $x \in\left[x_{0}\right]_{\widetilde{G}}$. Then, there is a path $x=z_{0}, z_{1}, \ldots, z_{l}=$ $x_{0}$ between $x$ and $x_{0}$. Since $f$ is a $b-(\varphi, G)$ contraction, then $\left(f z_{i-1}, f z_{i}\right) \in E(G)$ for all $i=1,2, \ldots, l$. Thus, $f x \in\left[f x_{0}\right]_{\widetilde{G}}=$ $\left[x_{0}\right]_{\widetilde{G}}$.

Suppose $(x, y) \in E\left(\widetilde{G}_{x_{0}}\right)$. Then, $(f x, f y) \in E(G)$, since $f$ is a $b-(\varphi, G)$ contraction. But $\left[x_{0}\right]_{\widetilde{G}}$ is $f$ invariant, so we conclude that $(f x, f y) \in E\left(\widetilde{G}_{x_{0}}\right)$. Condition (2) is satisfied automatically as $\widetilde{G}_{x_{0}}$ is a subgraph of $G$.

From now on, we assume that coefficient of $b$-comparison function is at least as large as the coefficient of $b$-metric $s$.

Lemma 12. Let $f: X \rightarrow X$ be a $b-(\varphi, G)$ contraction, where $\varphi: \mathbb{R}^{+} \rightarrow \mathbb{R}^{+}$is a $b$-comparison function. Then, given any $x \in X$ and $y \in[x]_{\widetilde{G}}$, two sequences $\left\{f^{n} x\right\}$ and $\left\{f^{n} y\right\}$ are equivalent.

Proof. Let $x \in X$, and let $y \in[x]_{\widetilde{G}}$; then there exists a path $\left\{x_{i}\right\}_{i=0}^{l}$ in $\widetilde{G}$ from $x$ to $y$ with $x_{0}=x, x_{l}=y$, and $\left(x_{i-1}, x_{i}\right) \in$ $E(\widetilde{G})$. From Proposition $11, f$ is a $b-(\varphi, \widetilde{G})$ contraction. So,

$$
\begin{aligned}
\left(f^{n} x_{i-1}, f^{n} x_{i}\right) & \in E(\widetilde{G}) \quad \text { implies } d\left(f^{n} x_{i-1}, f^{n} x_{i}\right) \\
& \leq \varphi\left(d\left(f^{n-1} x_{i-1}, f^{n-1} x_{i}\right)\right)
\end{aligned}
$$

for all $n \in \mathbb{N}$ and $i=0,1,2, \ldots, l$. Hence,

$$
\begin{gathered}
d\left(f^{n} x_{i-1}, f^{n} x_{i}\right) \leq \varphi^{n}\left(d\left(x_{i-1}, x_{i}\right)\right) \\
\forall n \in \mathbb{N}, \quad i=0,1,2, \ldots, l .
\end{gathered}
$$

We observe that $\left\{f^{n} x_{i}\right\}_{i=0}^{l}$ is a path in $\widetilde{G}$ from $f^{n} x$ to $f^{n} y$. Using (d3) Definition 1 and (4), we have

$$
\begin{aligned}
d\left(f^{n} x, f^{n} y\right) & \leq \sum_{i=1}^{l} s^{i} d\left(f^{n} x_{i-1}, f^{n} x_{i}\right) \\
& \leq \sum_{i=1}^{l} s^{i} \varphi^{n}\left(d\left(x_{i-1}, x_{i}\right)\right) .
\end{aligned}
$$

Letting $n \rightarrow \infty$, we obtain $d\left(f^{n} x, f^{n} y\right) \rightarrow 0$.

Proposition 13. Let $f$ be a $b-(\varphi, G)$ contraction, where $\varphi$ : $\mathbb{R}^{+} \rightarrow \mathbb{R}^{+}$is a b-comparison function. Suppose that there is $z_{0}$ in $X$ such that $f z_{0} \in\left[z_{0}\right]_{\widetilde{G}}$. Then, $\left\{f^{n} z_{0}\right\}$ is a Cauchy sequence in $X$.

Proof. Since $f z_{0} \in\left[z_{0}\right]_{\widetilde{G}}$, let $\left\{y_{i}\right\}_{i=0}^{r}$ be a path from $z_{0}$ to $f z_{0}$. Then using the same arguments as in Lemma 12, we arrive at

$$
d\left(f^{n} z_{0}, f^{n+1} z_{0}\right) \leq \sum_{i=1}^{r} s^{i} \varphi^{n}\left(d\left(y_{i-1}, y_{i}\right)\right), \quad \forall n \in \mathbb{N}
$$

Let $m>n \geq 1$, and then from above inequality; it follows for $p \geq 1$

$$
\begin{aligned}
d\left(f^{n} z_{0}, f^{n+p} z_{0}\right) & \\
\leq & s d\left(f^{n} z_{0}, f^{n+1} z_{0}\right)+s^{2} d\left(f^{n+1} z_{0}, f^{n+2} z_{0}\right) \\
& +\cdots+s^{p} d\left(f^{n+p-1} z_{0}, f^{n+p} z_{0}\right) \\
\leq & \frac{1}{s^{n-1}}\left[\sum_{j=n}^{n+p-1} s^{j} d\left(f^{j} z_{0}, f^{j+1} z_{0}\right)\right] \\
\leq & \frac{1}{s^{n-1}}\left[\sum_{i=1}^{r} s^{i} \sum_{j=n}^{n+p-1} s^{j} \varphi^{j}\left(d\left(y_{i-1}, y_{i}\right)\right)\right] .
\end{aligned}
$$

Denoting for each $i=1,2, \ldots, r$

$$
S_{n}^{i}=\sum_{k=0}^{n} s^{k} \varphi^{k}\left(d\left(y_{i-1}, y_{i}\right)\right), \quad n \geq 1,
$$

relation (7) becomes

$$
d\left(f^{n} z_{0}, f^{n+p} z_{0}\right) \leq \frac{1}{s^{n-1}}\left[\sum_{i=1}^{r} s^{i}\left[S_{n+p-1}^{i}-S_{n-1}^{i}\right]\right],
$$

since $\varphi$ is a $b$-comparison function, so that for each $i=$ $1,2, \ldots, r$,

$$
\sum_{k=0}^{\infty} s^{k} \varphi^{k}\left(d\left(y_{i-1}, y_{i}\right)\right)<\infty
$$

Then, corresponding to each $i$, there is a real number $S^{i}$ such that

$$
\lim _{n \rightarrow \infty} S_{n}^{i}=S^{i}
$$

In view of (11), relation (9) gives $d\left(f^{n} z_{0}, f^{n+p} z_{0}\right) \rightarrow 0$ as $n \rightarrow \infty$. Which shows that $\left\{f^{n} z_{0}\right\}$ is a Cauchy sequence in $X$.

Definition 14. Let $f: X \rightarrow X$, and let $y \in X$, and the sequence $\left\{f^{n} y\right\}$ in $X$ is such that $f^{n} y \rightarrow x^{*}$ with $\left(f^{n} y, f^{n+1} y\right) \in E(G)$ for $n \in \mathbb{N}$. One says that a graph $G$ is $\left(C_{f}\right)$-graph if there exists a subsequence $\left\{f^{n_{k}} y\right\}$ and a natural number $p$ such that $\left(f^{n_{k}} y, x^{*}\right) \in E(G)$ for all $k \geq p$ [23]. One says that a graph $G$ is $\left(H_{f}\right)$-graph if $f^{n} y \in\left[x^{*}\right]_{\widetilde{G}}$ for $n \geq 1$; then $r\left(f^{n} y, x^{*}\right) \rightarrow 0($ as $n \rightarrow \infty)$, where $r\left(f^{n} y, x^{*}\right)=$ $\sum_{i=1}^{M} s^{i} d\left(z_{i-1}, z_{i}\right) ;\left\{z_{i}\right\}_{i=0}^{M}$ is a path from $f^{n} y$ to $x^{*}$ in $\widetilde{G}$.

Obviously every $(C)$-graph is a $\left(C_{f}\right)$-graph for any selfmapping $f$ on $X$, but converse may not hold as shown in the following.

Example 15. Let $X=[0,1]$ with respect to $b$-metric $d(x, y)=$ $|x-y|^{2}$. Consider a graph $G$ consisting of $V(G)=X$ and $E(G)=\{(n /(n+1),(n+1) /(n+2)): n \in \mathbb{N}\} \cup\left\{\left(x / 2^{n}, x / 2^{n+1}\right):\right.$ $n \in \mathbb{N}, x \in[0,1]\} \cup\left\{\left(\left(x / 2^{2 n}\right), 0\right): n \in \mathbb{N}, x \in[0,1]\right\}$. Note that $G$ is not a $(C)$-graph as $n /(n+1) \rightarrow 1$. Define $f: X \rightarrow X$ as $f x=x / 2$. Then, $G$ is a $\left(C_{f}\right)$-graph, since $f^{n} x=x / 2^{n} \rightarrow 0$. 
Example 16. Let $X=\{1 / n: n \in \mathbb{N}\} \cup\{0\} \cup \mathbb{N}$ with respect to $b$-metric $d(x, y)=|x-y|^{2}$, and let $I$ be identity map on $X$. Consider a graph $G_{2}$ consisting of $V\left(G_{2}\right)=X$ and

$$
\begin{aligned}
E\left(G_{2}\right)=\{ & \left(\frac{1}{n}, \frac{1}{n+1}\right),\left(\frac{1}{n+1}, n\right),(n, 0), \\
& \left.\left(\frac{1}{5 n}, 0\right) ; n \in \mathbb{N}\right\},
\end{aligned}
$$

since $x_{n}=1 / n \rightarrow 0$ as $n \rightarrow \infty$. We note that $G_{2}$ is a $\left(C_{I}\right)$ graph, but $r\left(x_{n}, 0\right)=2|(1 / n)-(1 /(n+1))|^{2}+2^{2} \mid(1 /(n+1))-$ $\left.n\right|^{2}+2^{2} n^{2} \nrightarrow 0$ as $n \rightarrow \infty$. Thus, $G_{2}$ is not an $\left(H_{I}\right)$-graph.

Example 17. Let $X=\{1 / n: n \in \mathbb{N}\} \cup\{\sqrt{5} / n: n \in \mathbb{N}\} \cup\{0\}$ with respect to $b$-metric $d(x, y)=|x-y|^{2}$, and let $I$ be identity map on $X$. Consider a graph $G_{3}$ consisting of $V\left(G_{3}\right)=X$ and

$$
E\left(G_{3}\right)=\left\{\left(\frac{1}{n}, \frac{1}{n+1}\right),\left(\frac{1}{n+1}, \frac{\sqrt{5}}{n}\right),\left(\frac{\sqrt{5}}{n}, 0\right) ; n \in \mathbb{N}\right\},
$$

since $x_{n}=1 / n \rightarrow 0$ as $n \rightarrow \infty$. Clearly $G_{3}$ is not a $\left(C_{I}\right)$ graph, but it is easy to verify that $G_{3}$ is an $\left(H_{I}\right)$-graph.

Above examples show that for a given $f$ notions, $\left(C_{f}\right)$ graph and $\left(H_{f}\right)$-graph are independent even if $f$ is identity map.

Theorem 18. Let $(X, d)$ be a complete b-metric space, and let $f$ be $a b-(\varphi, G)$ contraction, where $\varphi$ is $b$-comparison function. Assume that $d$ is continuous and there is $z_{0}$ in $X$ for which $\left(z_{0}, f z_{0}\right)$ is an edge in $\widetilde{G}$. Then, the following assertions hold.

(1) If $G$ is a $\left(C_{f}\right)$-graph, then $f$ has a unique fixed point $t \in\left[z_{0}\right]_{\widetilde{G}}$, and for any $y \in\left[z_{0}\right]_{\widetilde{G}}, f^{n} y \rightarrow t$. Further, if $G$ is weakly connected, then $f$ is Picard operator.

(2) If $G$ is weakly connected $\left(H_{f}\right)$-graph, then $f$ has a unique fixed point $t \in X$ and for any $y \in X, f^{n} y \rightarrow t$.

Proof. (1) It follows from Proposition 13 that $\left\{f^{n} z_{0}\right\}$ is a Cauchy sequence in $X$. Since $X$ is complete, there exists $t \in X$ such that $f^{n} z_{0} \rightarrow t$. Since $\left(f^{n} z_{0}, f^{n+1} z_{0}\right) \in E(G)$, for all $n \in \mathbb{N}$, and $G$ is a $\left(C_{f}\right)$ graphs, there exists a subsequence $\left\{f^{n_{k}} z_{0}\right\}$ of $\left\{f^{n} z_{0}\right\}$ and $p \in \mathbb{N}$ such that $\left(f^{n_{k}} z_{0}, t\right) \in E(G)$ for all $k \geq p$. Observe that $\left(z_{0}, f z_{0}, f^{2} z_{0}, \ldots, f^{n_{1}} z_{0}, \ldots, f^{n_{p}} z_{0}, t\right)$ is a path in $\widetilde{G}$. Therefore, $t \in\left[z_{0}\right]_{\widetilde{G}}$. From (2), we get

$$
d\left(f^{n_{k}+1} z_{0}, f t\right) \leq \varphi\left(d\left(f^{n_{k}} z_{0}, t\right)\right)<d\left(f^{n_{k}} z_{0}, t\right) \quad \forall k \geq n_{0} .
$$

Letting $k \rightarrow \infty$, we obtain $\lim _{k \rightarrow \infty} f^{n_{k}+1} z_{0}=f t$, as $d$ is continuous. Since $\left\{f^{n_{k}} z_{0}\right\}$ is a subsequence of $\left\{f^{n} z_{0}\right\}$, we conclude that $f t=t$. Finally, if $y \in\left[z_{0}\right]_{\widetilde{G}}$, it follows from Lemma 12 that $f^{n} y \rightarrow t$.

(2) Let $G$ be weakly connected $\left(H_{f}\right)$-graph. From Proposition 13, $f^{n} z_{0} \rightarrow t \in X$, and then $r\left(f^{n} z_{0}, t\right) \rightarrow 0$ as $n \rightarrow \infty$. Now, for each $n \in \mathbb{N}$, let $\left\{y_{i}^{n}\right\} ; i=0,1, \ldots, M_{n}$ be a path from $f^{n} z_{0}$ to $t$ with $y_{0}=t$ and $y_{M_{n}}^{n}=f^{n} z_{0}$ in $\widetilde{G}$; then

$$
\begin{aligned}
d(t, f t) & \leq s\left[d\left(t, f^{n+1} z_{0}\right)+d\left(f^{n+1} z_{0}, f t\right)\right] \\
& \leq s\left[d\left(t, f^{n+1} z_{0}\right)+\sum_{i=1}^{M_{n}} s^{i} d\left(f y_{i-1}^{n}, f y_{i}^{n}\right)\right] \\
& \leq s\left[d\left(t, f^{n+1} z_{0}\right)+\sum_{i=1}^{M_{n}} s^{i} \varphi\left(d\left(y_{i-1}^{n}, y_{i}^{n}\right)\right)\right] \\
& <s\left[d\left(t, f^{n+1} z_{0}\right)+\sum_{i=1}^{M_{n}} s^{i} d\left(y_{i-1}^{n}, y_{i}^{n}\right)\right] \\
& =s\left[d\left(t, f^{n+1} z_{0}\right)+r\left(f^{n} z_{0}, t\right)\right] .
\end{aligned}
$$

Letting $n \rightarrow \infty$, the above inequality yields $f t=t$. Let $y \in$ $\left[z_{0}\right]_{\widetilde{G}}:=X$ be arbitrary; then from Lemma 12 and Remark 10, it is easily seen that $f^{n} y \rightarrow t$.

The following example shows that the condition of $\left(C_{f}\right)$ or $\left(H_{f}\right)$-graph in the hypothesis of Theorem 18 can not be dropped.

Example 19. Let $X=[0,1]$, let $d(x, y)=|x-y|^{2}$, and let $f x=$ $x / 2$ for all $x \in(0,1]$ and $f 0=1 / 2$. Then, $(X, d)$ is a complete $b$-metric space with $s=2$. Further, $d$ is continuous, and $f$ is a $b$ - $\left(\varphi, G_{1}\right)$ contraction (with $\left.\varphi(t)=t / 4\right)$, where $V\left(G_{1}\right)=X$ and $E\left(G_{1}\right)=\{(x, y) \in(0,1] \times(0,1] ; x \geq y\} \cup\{(0,0),(0,1)\}$. Note that $G_{1}$ is weakly connected, but $f$ has no fixed point in $\left[z_{0}\right]_{\widetilde{G}_{1}}=X$. Observe that $G_{1}$ is not a $\left(C_{f}\right)$-graph because the sequence $f^{n} x=x / 2^{n} \rightarrow 0$ for $x \in(0,1]$ and $\left(f^{n} x, f^{n+1} x\right) \epsilon$ $E\left(G_{1}\right) ; n \in \mathbb{N}$, but it does not contain any subsequence such that $\left(x_{n_{k}}, 0\right) \in E\left(G_{1}\right)$. Also, we note that for any fixed $x \in$ $(0,1], r\left(f^{n} x, 0\right)=2\left[\left|x / 2^{n}-1\right|^{2}+|1-0|^{2}\right] \nrightarrow 0$ as $n \rightarrow \infty$.

Definition 20. Let $(X, d)$ be a $b$-metric space. A mapping $f$ : $X \rightarrow X$ is called orbitally continuous if for all $x, y \in X$ and any sequence $\left\{k_{n}\right\}_{n \in \mathbb{N}}$ of positive integers, $f^{k_{n}} x \rightarrow y$ implies $f\left(f^{k_{n}} x\right) \rightarrow f y$ as $n \rightarrow \infty$. A mapping $f: X \rightarrow X$ is called orbitally $G$-continuous if for all $x, y \in X$ and any sequence $\left\{k_{n}\right\}_{n \in \mathbb{N}}$ of positive integers, $f^{k_{n}} x \rightarrow y$ and $\left(f^{k_{n}} x, f^{k_{n}+1} x\right) \in$ $E(G)$ for all $n \in \mathbb{N}$ imply $f\left(f^{k_{n}} x\right) \rightarrow f y$.

Theorem 21. Let $(X, d)$ be a complete b-metric space, and let $f$ be a $b-(\varphi, G)$ contraction, where $\varphi$ is a b-comparison function. Assume that $d$ is continuous, $f$ is orbitally G-continuous, and there is $z_{0}$ in $X$ for which $\left(z_{0}, f z_{0}\right)$ is an edge in $G$. Then, $f$ has a fixed point $t \in X$. Moreover, for any $y \in\left[z_{0}\right]_{\widetilde{G}}, f^{n} y \rightarrow t$.

Proof. It follows from Proposition 13 that $\left\{f^{n} z_{0}\right\}$ is a Cauchy sequence in $(X, d)$. Since $X$ is complete, there exists $t \in X$ such that $\lim _{n \rightarrow \infty} f^{n} z_{0}=t$. Since $\left(f^{n} z_{0}, f^{n+1} z_{0}\right) \in E(G)$ for all $n \in \mathbb{N}, f$ is orbitally $G$-continuous. Therefore, continuity of $d$ implies that $f t=t$. Let $y \in\left[z_{0}\right]_{\widetilde{G}}$ be arbitrary; then it follows from Lemma 12 that $\lim _{n \rightarrow \infty} f^{n} y=t$. 
Slightly strengthening the continuity condition on $f$, our next theorem deals with the graph $G$ which may fail to have the property that there is $z_{0}$ in $X$ for which $\left(z_{0}, f z_{0}\right)$ is an edge in $G$.

Theorem 22. Let $(X, d)$ be a complete b-metric space, and let $f$ be a $b-(\varphi, G)$ contraction, where $\varphi$ is a b-comparison function. Assume that $d$ is continuous, $f$ is orbitally continuous, and there is $z_{0}$ in $X$ for which $f z_{0} \in\left[z_{0}\right]_{\widetilde{G}}$. Then, for any $y \in\left[z_{0}\right]_{\widetilde{G}}$, $f^{n} y \rightarrow t \in X$, where $t$ is a fixed point of $f$.

Proof. It follows from Proposition 13 that $\left\{f^{n} z_{0}\right\}$ is a Cauchy sequence in $X$. Since $X$ is complete, there exists $t \in X$ such that $f^{n} z_{0} \rightarrow t$. Since $f$ is orbitally continuous, then $\lim _{n \rightarrow \infty} f f^{n} z_{0}=f t$ which yields $f t=t$. Let $y \in\left[z_{0}\right]_{\widetilde{G}}$ be arbitrary; then from Lemma $12, \lim _{n \rightarrow \infty} f^{n} y=t$.

Remark 23. In addition to the hypothesis of Theorems 21 and 22 , if we assume that $G$ is weakly connected, then $f$ will become Picard operator [11] on $X$.

Remark 24. Theorem 18 generalizes/extends claims $4^{0}$ and $5^{0}$ of [10, Theorem 3.2] and [7, Theorem 4(1)]. Theorem 21 generalizes claims $2^{0}$ and $3^{0}$ of [10, Theorem 3.3]. Theorem 22 generalizes claims $2^{0}$ and $3^{0}$ of [10, Theorem 3.4], and thus generalizes extends results of Nieto and Rodríguez-López [24, Theorems 2.1 and 2.3], Petrusel and Rus [11, Theorem 4.3], and Ran and Reurings [25, Theorem 2.1]. We mention here that Theorem 18 can not be improved using comparison function instead of b-comparison function (see, Gwóźdź-Łukawska and Jachymski [26, Example 2]).

We observe that Theorem 22 can be used to extend famous fixed point theorem of Edelstein to the case of $b$-metric space. We need to define notion of $\epsilon$-chainable property for $b$-metric space.

Definition 25. A $b$-metric space $(X, d)$ is said to be $\epsilon$ chainable, for some $\epsilon>0$, if for $x, y \in X$ there exist $x_{i} \in X ; i=$ $0,1,2, \ldots, l$ with $x_{0}=x, x_{l}=y$ such that $d\left(x_{i-1}, x_{i}\right)<\epsilon$ for $i=1,2, \ldots, l$.

Corollary 26. Let $(X, d)$ be a complete $\epsilon$-chainable b-metric space. Assume that $d$ is continuous, and there exists $a b$ comparison function $\varphi: \mathbb{R}^{+} \rightarrow \mathbb{R}^{+}$such that $f: X \rightarrow X$ satisfying,

$$
d(x, y)<\epsilon \quad \text { implies } d(f x, f y) \leq \varphi(d(x, y)),
$$

for all $x, y \in X$. Then, $f$ is a Picard operator.

Proof. Consider a graph $G$ consisting of $V(G):=X$ and $(x, y) \in E(G)$ if and only if $d(x, y)<\epsilon$. Since $X$ is $\epsilon$-chainable, $G$ is weakly connected. Let $(x, y) \in E(G)$, and from (16), we have

$$
d(f x, f y) \leq \varphi(d(x, y))<d(x, y)<\epsilon .
$$

Then, $d(f x, f y) \in E(G)$. Therefore, in view of (16), $f$ is $b$ $(\varphi, G)$ contraction. Further, (16) implies that $f$ is continuous. Now, the conclusion follows by using Theorem 22 .
Now, we establish a fixed point theorem using a general contractive condition.

Theorem 27. Let $(X, d)$ be a complete b-metric space, and let $G$ be a $\left(C_{f}\right)$-graph in $X \times X$ such that $V(G)=X$ and $f: X \rightarrow$ $X$ is an edge-preserving mapping. Assume that $d$ is continuous and there exist $\alpha, \beta, \gamma \geq 0$ with $s \alpha+(s+1) \beta+s(s+1) \gamma<1$ and for all $(x, y) \in E(G)$

$$
\begin{aligned}
d(f x, f y) \leq & \alpha d(x, y)+\beta[d(x, f x)+d(y, f y)] \\
& +\gamma[d(x, f y)+d(y, f x)] .
\end{aligned}
$$

If there is $z_{0}$ in $X$ for which $\left(z_{0}, f z_{0}\right)$ is an edge in $G$, then $f$ has a fixed point in $\left[z_{0}\right]_{\widetilde{G}}$.

Proof. Since $f$ is edge-preserving, then $\left(f^{n} z_{0}, f^{n+1} z_{0}\right) \in$ $E(G)$ for all $n \in \mathbb{N}$. From (18) and using (d3), it follows that

$$
\begin{aligned}
d\left(f^{n} z_{0},\right. & \left.f^{n+1} z_{0}\right) \\
\leq & \alpha d\left(f^{n-1} z_{0}, f^{n} z_{0}\right) \\
& +\beta\left[d\left(f^{n-1} z_{0}, f^{n} z_{0}\right)+d\left(f^{n} z_{0}, f^{n+1} z_{0}\right)\right] \\
& +\gamma s\left[d\left(f^{n-1} z_{0}, f^{n} z_{0}\right)+d\left(f^{n} z_{0}, f^{n+1} z_{0}\right)\right] .
\end{aligned}
$$

On rearranging,

$$
d\left(f^{n} z_{0}, f^{n+1} z_{0}\right) \leq\left[\frac{\alpha+\beta+\gamma s}{1-\beta-\gamma s}\right] d\left(f^{n-1} z_{0}, f^{n} z_{0}\right) .
$$

Repeating iteratively, we have

$$
d\left(f^{n} z_{0}, f^{n+1} z_{0}\right) \leq\left[\frac{\alpha+\beta+\gamma s}{1-\beta-\gamma s}\right]^{n} d\left(z_{0}, f z_{0}\right) .
$$

For $m>n \geq 1$ and using (d3) Definition 1, we have

$$
\begin{aligned}
d\left(f^{n} z_{0}, f^{m} z_{0}\right) & \\
\leq & s d\left(f^{n} z_{0}, f^{n+1} z_{0}\right)+s^{2} d\left(f^{n+1} z_{0}, f^{n+2} z_{0}\right) \\
& +\cdots+s^{m-n} d\left(f^{m-1} z_{0}, f^{m} z_{0}\right) \\
\leq & \frac{1}{s^{n-1}} d\left(z_{0}, f z_{0}\right) \sum_{j=n}^{m-1} s^{j}\left[\frac{\alpha+\beta+\gamma s}{1-\beta-\gamma s}\right]^{j} \\
< & \frac{1}{s^{n-1}} d\left(z_{0}, f z_{0}\right) \sum_{j=n}^{\infty} s^{j}\left[\frac{\alpha+\beta+\gamma s}{1-\beta-\gamma s}\right]^{j} .
\end{aligned}
$$

Since $s[(\alpha+\beta+\gamma s) /(1-\beta-\gamma s)]<1$, then $\left\{f^{n} z_{0}\right\}$ is a Cauchy sequence in $X$. By completeness of $X$, the sequence $\left\{f^{n} z_{0}\right\}$ converges to $t \in X$. Since $G$ is a $\left(C_{f}\right)$-graph, there exists a subsequence $\left\{f^{n_{k}} z_{0}\right\}$ and a natural number $p$ such that 
$\left(f^{n_{k}} z_{0}, t\right) \in E(G)$ for all $k \geq p$. Using (18) for all $k \geq p$, we have

$$
\begin{aligned}
& d\left(f^{n_{k}+1} z_{0}, f t\right) \\
& \leq \quad \alpha d\left(f^{n_{k}} z_{0}, t\right)+\beta\left[d\left(f^{n_{k}} z_{0}, f^{n_{k}+1} z_{0}\right)+d(t, f t)\right] \\
& \quad+\gamma\left[d\left(f^{n_{k}} z_{0}, f t\right)+d\left(t, f^{n_{k}+1} z_{0}\right)\right] .
\end{aligned}
$$

Since the $b$-metric $d$ is continuous and $\beta+\gamma<1$, letting $k \rightarrow \infty$ inequality (23) yields $f t=t$. Also note that $\left(z_{0}, f z_{0}, f^{2} z_{0}, \ldots, f^{n_{1}} z_{0}, \ldots, f^{n_{p}}, t\right)$ is a path in $G$ and hence in $\widetilde{G}$, therefore $t \in\left[z_{0}\right]_{\widetilde{G}}$.

We note that Theorem 27 does not guarantee the uniqueness of fixed point, but this can be accomplished under some assumptions as in the following theorem.

Theorem 28. In addition to the hypothesis of Theorem 27, one further assumes that if $\alpha+2 s \beta+2 \gamma<1$ for the same set of $\alpha, \beta, \gamma \geq 0$ and for any two fixed points $t_{1}, t_{2} \exists z \in X$ such that $\left(t_{1}, z\right)$ and $\left(t_{2}, z\right) \in E(G)$. Then, $f$ has a unique fixed point.

Proof. Let $t_{1}$ and $t_{2}$ be two fixed points of $f$, and then there exists $z \in X$ such that $\left(t_{1}, z\right),\left(t_{2}, z\right) \in E(G)$. By induction, we have $\left(t_{1}, f^{n} z\right),\left(t_{2}, f^{n} z\right) \in E(G)$ for all $n=0,1, \ldots$. From (18), we have

$$
\begin{aligned}
d\left(t_{1}, f^{n} z\right) \leq & \alpha d\left(t_{1}, f^{n-1} z\right)+\beta d\left(f^{n-1} z, f^{n} z\right) \\
& +\gamma\left[d\left(t_{1}, f^{n} z\right)+d\left(f^{n-1} z, t_{1}\right)\right] \\
\leq & \alpha d\left(t_{1}, f^{n-1} z\right)+\beta s\left[d\left(f^{n-1} z, t_{1}\right)+d\left(t_{1}, f^{n} z\right)\right] \\
& +\gamma\left[d\left(t_{1}, f^{n} z\right)+d\left(f^{n-1} z, t_{1}\right)\right] .
\end{aligned}
$$

On rearranging,

$$
d\left(t_{1}, f^{n} z\right) \leq\left[\frac{\alpha+s \beta+\gamma}{1-s \beta-\gamma}\right] d\left(t_{1}, f^{n-1} z\right), \quad \forall n=1,2, \ldots
$$

Continuing recursively, (25) gives

$$
d\left(t_{1}, f^{n} z\right) \leq\left[\frac{\alpha+s \beta+\gamma}{1-s \beta-\gamma}\right]^{n} d\left(t_{1}, z\right)
$$

Since $[(\alpha+s \beta+\gamma) /(1-s \beta-\gamma)]<1$, then $\lim _{n \rightarrow \infty} d\left(t_{1}, f^{n} z\right)=$ 0 . Similarly, one can show that $\lim _{n \rightarrow \infty} d\left(t_{2}, f^{n} z\right)=0$, which by using (d3) Definition 1 infers that $d\left(t_{1}, t_{2}\right)=0$.

Suppose that $(X, \preceq)$ is a partially ordered set. Consider graph $G_{2}$ consisting of $E\left(G_{2}\right)=\{(x, y) \in X \times X: x \preceq$ $y$ or $y \preceq x\}$, and $V\left(G_{2}\right)$ coincides with $X$. We note that if a self-mapping $f$ is monotone with respect to the order, then, for graph $G_{2}$, it is obvious that $f$ is edge-preserving, or equivalently we can say that $f$ maps comparable elements onto comparable elements.

Following corollaries are the direct consequences of Theorem 28.
Corollary 29. Let $(X, d)$ be a complete metric space, where $X$ is partially ordered set with respect to $\preceq$. Let $f: X \rightarrow X$ be nondecreasing (or nonincreasing) with respect to $\preceq$. Assume that there exists $\alpha, \beta, \gamma \geq 0$ with $\alpha+2 \beta+2 \gamma<1$ such that,

$$
\begin{aligned}
d(f x, f y) \leq & \alpha d(x, y)+\beta[d(x, f x)+d(y, f y)] \\
& +\gamma[d(x, f y)+d(y, f x)]
\end{aligned}
$$

for all comparable $x, y \in X$. If the following conditions hold:

(i) there exists $x_{0} \in X$ such that $x_{0} \preceq f x_{0}$,

(ii) for nondecreasing (or nonincreasing) sequence $\left\{x_{n}\right\} \rightarrow$ $x \in X$, there exists a subsequence $\left\{x_{n_{k}}\right\}$ such that $x_{n_{k}} \preceq$ $x$, for all $k$.

Then, $f$ has a fixed point. Moreover, if for all $x, y \in X$ there exists $z \in X$ such that $x \preceq z$ and $y \preceq z$, then the fixed point is unique.

Corollary 30. Let $(X, d)$ be a complete metric space, where $X$ is partially ordered set with respect to $\preceq$. Let $f: X \rightarrow X$ be nondecreasing (or nonincreasing) with respect to $\preceq$. Assume that there exists a constant $0<c<1 / 2$ such that

$$
d(f x, f y) \leq c[d(x, f x)+d(y, f y)]
$$

for all comparable $x, y \in X$. If the following conditions hold:

(i) there exists $x_{0} \in X$ such that $x_{0} \preceq f x_{0}$,

(ii) for nondecreasing (or nonincreasing) sequence $\left\{x_{n}\right\} \rightarrow$ $x \in X$, there exists a subsequence $\left\{x_{n_{k}}\right\}$ such that $x_{n_{k}} \preceq$ $x$, for all $k$.

Then, $f$ has a fixed point. Moreover, if for all $x, y \in X$ there exists $z \in X$ such that $x \preceq z$ and $y \preceq z$, then the fixed point is unique.

Corollary 31. Let $(X, d)$ be a complete metric space, where $X$ is partially ordered set with respect to $\preceq$. Let $f: X \rightarrow X$ be nondecreasing (or nonincreasing) with respect to $\preceq$. Assume that there exists a constant $0<c<1 / 2$ such that

$$
d(f x, f y) \leq c[d(x, f y)+d(y, f x)]
$$

for all comparable $x, y \in X$. If the following conditions hold:

(i) there exists $x_{0} \in X$ such that $x_{0} \preceq f x_{0}$,

(ii) for nondecreasing (or nonincreasing) sequence $\left\{x_{n}\right\} \rightarrow$ $x \in X$, there exists a subsequence $\left\{x_{n_{k}}\right\}$ such that $x_{n_{k}} \preceq$ $x$, for all $k$.

Then, $f$ has a fixed point. Moreover, if for all $x, y \in X$ there exists $z \in X$ such that $x \preceq z$ and $y \preceq z$, then the fixed point is unique.

Remark 32. We note that in Theorem 27, the condition "there

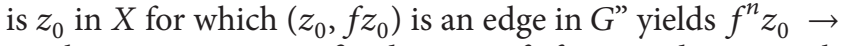
$t$, where $t \in X$ is a fixed point of $f$. Consider a graph $G:=(X, X \times X)$. For such graph under the assumptions of Theorems 27 and 28, it infers that $f$ is a Picard operator. Thus, many standard fixed point theorems can be easily deduced from Theorem 28 as follows. 
Corollary 33 (Hardy and Rogers [27]). Let $(X, d)$ be a complete metric space, and let $f: X \rightarrow X$. Suppose that there exists constants $\alpha, \beta, \gamma \geq 0$ such that

$$
\begin{aligned}
d(f x, f y) \leq & \alpha d(x, y)+\beta[d(x, f x)+d(y, f y)] \\
& +\gamma[d(x, f y)+d(y, f x)],
\end{aligned}
$$

for all $x, y \in X$, where $\alpha+2 \beta+2 \gamma<1$; then $f$ has a unique fixed point in $X$.

Corollary 34 (Kannan [28]). Let $(X, d)$ be a complete metric space, and let $f: X \rightarrow X$. Suppose that there exists a constants c such that

$$
d(f x, f y) \leq c[d(x, f x)+d(y, f y)]
$$

for all $x, y \in X$, where $0<c<1 / 2$; then $f$ is Picard operator.

Corollary 35 (Chatterjea [29]). Let $(X, d)$ be a complete metric space, and let $f: X \rightarrow X$. Suppose that there exists a constants $c$ such that

$$
d(f x, f y) \leq c[d(x, f y)+d(y, f x)],
$$

for all $x, y \in X$, where $0<c<1 / 2$; then $f$ is Picard operator.

\section{Applications}

Let $X$ be a nonempty set, let $m$ be a positive integer, $\left\{X_{i}\right\}_{i=1}^{m}$ be nonempty closed subsets of $X$, and let $f: \bigcup_{i=1}^{m} X_{i} \rightarrow$ $\bigcup_{i=1}^{m} X_{i}$ be an operator. Then, $X:=\bigcup_{i=1}^{m} X_{i}$ is known as cyclic representation of $X$ w.r.t. $f$ if

$$
f\left(X_{1}\right) \subset X_{2}, \ldots, f\left(X_{m-1}\right) \subset X_{m}, \quad f\left(X_{m}\right) \subset X_{1},
$$

and operator $f$ is known as cyclic operator [30].

Theorem 36. Let $(X, d)$ be complete b-metric space such that $d$ is continuous functional on $X \times X$. Let $m$ be positive integer, let $\left\{X_{i}\right\}_{i=1}^{m}$ be nonempty closed subsets of $X$, let $Y:=\bigcup_{i=1}^{m} X_{i}$, $\varphi: \mathbb{R}^{+} \rightarrow \mathbb{R}^{+}$be a b-comparison function, and let $f: Y \rightarrow$ $Y$. Further, suppose that

(i) $\bigcup_{i=1}^{m} X_{i}$ is cyclic representation of $Y$ w.r.t. $f$,

(ii) $d(f x, f y) \leq \varphi(d(x, y))$ whenever $x \in X_{i}, y \in X_{i+1}$, where $X_{m+1}=X_{1}$.

Then, $f$ has a unique fixed point $t \in \bigcap_{i=1}^{m} X_{i}$ and $f^{n} y \rightarrow t$ for any $y \in \bigcup_{i=1}^{m} X_{i}$.

Proof. We note that $(Y, d)$ is complete $b$-metric space. Let us consider a graph $G$ consisting of $V(G):=Y$ and $E(G):=$ $\Delta \cup\left\{(x, y) \in Y \times Y: x \in X_{i}, y \in X_{i+1} ; i=1, \ldots, m\right\}$. By (i), it follows that $f$ preserves edges. Now, let $f^{n} x \rightarrow x^{*}$ in $Y$ such that $\left(f^{n} x, f^{n+1} x\right) \in E(G)$ for all $n \geq 1$; then in view of (33), sequence $\left\{f^{n} x\right\}$ has infinitely many terms in each $X_{i}$ so that one can easily extract a subsequence of $\left\{f^{n} x\right\}$ converging to $x^{*}$ in each $X_{i}$. Since $X_{i}$ 's are closed, then $x^{*} \in \bigcap_{i=1}^{m} X_{i}$. Now, it is easy to form a subsequence $\left\{f^{n_{k}} x\right\}$ in some $X_{j}$, $j \in\{1, \ldots, m\}$ such that $\left(f^{n_{k}} x, x^{*}\right) \in E(G)$ for $k \geq 1$, and it indicates that $G$ is weakly connected $\left(C_{f}\right)$-graph, and thus conclusion follows from Theorem 18.
Remark 37 (see [31, Theorem 2.1(1)]). It can be deduced from Theorem 36 if $(X, d)$ is a metric space.

On the same lines as in proof of Theorem 36, we obtain the following consequence of Theorem 28 .

Theorem 38. Let $(X, d)$ be a complete b-metric space such that $d$ is continuous functional on $X \times X$. Let $m$ be positive integer, let $\left\{X_{i}\right\}_{i=1}^{m}$ be nonempty closed subsets of $X$, let $Y:=\bigcup_{i=1}^{m} X_{i}$, and let $f: Y \rightarrow Y$. Further, suppose that

(i) $\bigcup_{i=1}^{m} X_{i}$ is cyclic representation of $Y$ w.r.t. $f$,

(ii) there exist $\alpha, \beta, \gamma \geq 0$ with $s \alpha+s(s+1) \beta+s(s+1) \gamma<1$ such that

$$
\begin{aligned}
& d(f x, f y) \\
& \leq \alpha d(x, y)+\beta[d(x, f x)+d(y, f y)] \\
& \quad+\gamma[d(x, f y)+d(y, f x)]
\end{aligned}
$$

whenever $x \in X_{i}, y \in X_{i+1}$, where $X_{m+1}=X_{1}$.

Then, $f$ has a fixed point $t \in \bigcap_{i=1}^{m} X_{i}$.

Remark 39. [32, Theorem 7] and [33, Theorem 3.1] can be deduced from Theorem 38, but it does not ensure $f$ to be a Picard operator.

Remark 40. We note that in proof [32, Theorem 7], the author's argument to prove that is $G$ (as assumed in proof of Theorem 36), a (C)-graph is valid only if the terms of sequence $\left\{x_{n}\right\}$ are Picard iterations; otherwise it is void. For example, let $Y=\bigcup_{i=1}^{3} X_{i}$ where $X_{1}=\{1 / n: n$ is odd $\} \cup\{0\}$, $X_{2}=\{1 / n: n$ is even $\} \cup\{0\}, X_{3}=\{0\}$, and define $f: Y \rightarrow Y$ as $f x=x / 2 ; x \in Y \backslash X_{2}$ and $f x=0 ; x \in X_{2}$. We see that (33) is satisfied and $1 / n \rightarrow 0$, but $X_{3}$ does not contain infinitely many terms of $\left\{x_{n}\right\}$. In the following, we give the corrected argument to prove that $G$ is a $(C)$-graph.

Let $x_{n} \rightarrow x$ in $X$ such that $\left(x_{n}, x_{n+1}\right) \in E(G)$ for all $n \geq 1$. Keeping in mind construction of $G$, there exists at least one pair of closed sets $\left\{X_{j}, X_{j+1}\right\}$ for some $j \in\{1,2, \ldots, m\}$ such that both sets contain infinitely many terms of sequence $\left\{x_{n}\right\}$, since $X_{i}$ 's are closed so that $x \in X_{j} \cap X_{j+1}$ for some $j \in\{1, \ldots, m\}$, and thus one can easily extract a subsequence such that $\left(x_{n_{k}}, x\right) \in E(G)$ holds for $k \geq 1$.

Now, we establish an existence theorem for the solution of an integral equation as a consequence of our Theorem 18.

Theorem 41. Consider the integral equation

$$
x(t)=\int_{a}^{b} k(t, s, x(s)) d s+g(t), \quad t \in[a, b],
$$

where $k:[a, b] \times[a, b] \times \mathbb{R}^{n} \rightarrow \mathbb{R}^{n}$ and $g:[a, b] \rightarrow \mathbb{R}^{n}$ is continuous. Assume that

(i) $k(t, s, \cdot): \mathbb{R}^{n} \rightarrow \mathbb{R}^{n}$ is nondecreasing for each $t, s \in$ $[a, b]$,

(ii) there exists a (c)-comparison function $\varphi: \mathbb{R}^{+} \rightarrow \mathbb{R}^{+}$ and a continuous function $p:[a, b] \times[a, b] \rightarrow \mathbb{R}^{+}$ 
such that $|k(t, s, x(s))-k(t, s, y(s))| \leq p(t, s) \varphi(\mid x(s)-$ $y(s) \mid)$ for each $t, s \in[a, b]$ and $x \leq y$ (i.e., $x(t) \leq y(t)$ for all $t \in[a, b])$,

(iii) $\sup _{t \in[a, b]} \int_{a}^{b} p(t, s) d s \leq 1$,

(iv) there exists $x_{0} \in C\left([a, b], \mathbb{R}^{n}\right)$ such that $x_{0}(t) \leq$ $\int_{a}^{b} k\left(t, s, x_{0}(s)\right) d s+g(t)$ for all $t \in[a, b]$.

Then, the integral equation (35) has a unique solution in the set $\left\{x \in C\left([a, b], \mathbb{R}^{n}\right): x(t) \leq x_{0}(t)\right.$ or $x(t) \geq x_{0}(t)$, for all $t \in[a, b]\}$.

Proof. Let $\left(X,\|\cdot\|_{\infty}\right)$, where $X=C\left([a, b], \mathbb{R}^{n}\right)$, and define a mapping $T: C\left([a, b], \mathbb{R}^{n}\right) \rightarrow C\left([a, b], \mathbb{R}^{n}\right)$ by

$$
T x(t)=\int_{a}^{b} k(t, s, x(s)) d s+g(t), \quad t \in[a, b] .
$$

Consider a graph $G$ consisting of $V(G):=X$ and $E(G)=$ $\{(x, y) \in X \times X: x(t) \leq y(t)$ for all $t \in[a, b]\}$. From property (i), we observe that the mapping $T$ is nondecreasing, thus $T$ preserves edges. Furthermore, $G$ is a $(C)$-graph; that is, for every nondecreasing sequence $\left\{x_{n}\right\} \subset X$ which converges to some $z \in X$; then $x_{n}(t) \leq z(t)$ for all $t \in[a, b]$. Now, for every $x, y \in X$ with $(x, y) \in E(G)$, we have

$$
\begin{aligned}
\mid T x(t) & -T y(t) \mid \\
\leq & \int_{a}^{b}|k(t, s, x(s))-k(t, s, y(s))| d s \\
\leq & \int_{a}^{b} p(t, s) \varphi(|x(s)-y(s)|) d s \\
\leq & \varphi\left(\|x-y\|_{\infty}\right) \int_{a}^{b} p(t, s) d s .
\end{aligned}
$$

Hence, $d(T x, T y) \leq \varphi(d(x, y))$. From (iv), we have $\left(x_{0}, T x_{0}\right) \in E(G)$, so that $\left[x_{0}\right]_{\widetilde{G}}=\left\{x \in C\left([a, b], \mathbb{R}^{n}\right): x(t) \leq\right.$ $x_{0}(t)$ or $x(t) \geq x_{0}(t)$ for all $\left.t \in[a, b]\right\}$. The conclusion follows from Theorem 18 .

Note that Theorem 41 specifies region of solution which invokes the novelty of our result.

\section{References}

[1] I. A. Bakhtin, "The contraction mapping principle in almost metric spaces," Journal of Functional Analysis, vol. 30, pp. 2637, 1989.

[2] J. Heinonen, Lectures on Analysis on Metric Spaces, Springer, New York, NY, USA, 2001.

[3] N. Bourbaki, Topologie Generale, Herman, Paris, France, 1974.

[4] S. Czerwik, "Nonlinear set-valued contraction mappings in b-metric spaces," Atti del Seminario Matematico e Fisico dell'Università di Modena, vol. 46, no. 2, pp. 263-276, 1998.

[5] M. Bota, A. Molnár, and C. Varga, "On Ekeland's variational principle in $b$-metric spaces," Fixed Point Theory, vol. 12, no. 1, pp. 21-28, 2011.
[6] M. F. Bota-Boriceanu and A. Petruşel, "Ulam-Hyers stability for operatorial equations," Analele Stiintifice ale Universitatii "Al. I. Cuza" din Iasi, vol. 57, supplement 1, pp. 65-74, 2011.

[7] M. Păcurar, "A fixed point result for $\varphi$-contractions on $b$-metric spaces without the boundedness assumption," Polytechnica Posnaniensis. Institutum Mathematicum. Fasciculi Mathematici, no. 43, pp. 127-137, 2010.

[8] V. Berinde, "Sequences of operators and fixed points in quasimetric spaces," Studia Universitatis Babeş-Bolyai, vol. 41, no. 4, pp. 23-27, 1996.

[9] S. Banach, "Sur les operations dans les ensembles abstraits et leur application aux equations integrales," Fundamenta Mathematicae, vol. 3, pp. 133-181, 1922.

[10] J. Jachymski, "The contraction principle for mappings on a metric space with a graph," Proceedings of the American Mathematical Society, vol. 136, no. 4, pp. 1359-1373, 2008.

[11] A. Petruşel and I. A. Rus, "Fixed point theorems in ordered 1spaces," Proceedings of the American Mathematical Society, vol. 134, no. 2, pp. 411-418, 2006.

[12] J. Matkowski, "Integrable solutions of functional equations," Dissertationes Mathematicae, vol. 127, p. 68, 1975.

[13] D. O'Regan and A. Petruşel, "Fixed point theorems for generalized contractions in ordered metric spaces," Journal of Mathematical Analysis and Applications, vol. 341, no. 2, pp. 12411252, 2008.

[14] R. P. Agarwal, M. A. El-Gebeily, and D. O’Regan, “Generalized contractions in partially ordered metric spaces," Applicable Analysis, vol. 87, no. 1, pp. 109-116, 2008.

[15] I. A. Rus, Generalized Contractions and Applications, Cluj University Press, Cluj-Napoca, Romania, 2001.

[16] V. Berinde, Contractii Generalizate si Aplicatii, vol. 22, Editura Cub Press, Baia Mare, Romania, 1997.

[17] V. Berinde, "Generalized contractions in quasimetric spaces," Seminar on Fixed Point Theory, vol. 3, pp. 3-9, 1993.

[18] T. P. Petru and M. Boriceanu, "Fixed point results for generalized $\varphi$-contraction on a set with two metrics," Topological Methods in Nonlinear Analysis, vol. 33, no. 2, pp. 315-326, 2009.

[19] R. P. Agarwal, M. A. Alghamdi, and N. Shahzad, "Fixed point theory for cyclic generalized contractions in partial metric spaces," Fixed Point Theory and Applications, vol. 2012, article 40, 11 pages, 2012.

[20] A. Amini-Harandi, "Coupled and tripled fixed point theory in partially ordered metric spaces with application to initial value problem," Mathematical and Computer Modelling, vol. 57, no. 910, pp. 2343-2348, 2012.

[21] H. K. Pathak and N. Shahzad, "Fixed points for generalized contractions and applications to control theory," Nonlinear Analysis: Theory, Methods \& Applications, vol. 68, no. 8, pp. 21812193, 2008.

[22] S. M. A. Aleomraninejad, Sh. Rezapour, and N. Shahzad, "Some fixed point results on a metric space with a graph," Topology and Its Applications, vol. 159, no. 3, pp. 659-663, 2012.

[23] M. Samreen and T. Kamran, "Fixed point theorems for integral G-contractions," Fixed Point Theory and Applications, vol. 2013, article 149, 2013.

[24] J. J. Nieto and R. Rodríguez-López, "Contractive mapping theorems in partially ordered sets and applications to ordinary differential equations," Order, vol. 22, no. 3, pp. 223-239, 2005.

[25] A. C. M. Ran and M. C. B. Reurings, "A fixed point theorem in partially ordered sets and some applications to matrix 
equations," Proceedings of the American Mathematical Society, vol. 132, no. 5, pp. 1435-1443, 2004.

[26] G. Gwóźdź-Łukawska and J. Jachymski, "IFS on a metric space with a graph structure and extensions of the Kelisky-Rivlin theorem," Journal of Mathematical Analysis and Applications, vol. 356, no. 2, pp. 453-463, 2009.

[27] G. E. Hardy and T. D. Rogers, "A generalization of a fixed point theorem of Reich," Canadian Mathematical Bulletin, vol. 16, pp. 201-206, 1973.

[28] R. Kannan, "Some results on fixed points," Bulletin of the Calcutta Mathematical Society, vol. 60, pp. 71-76, 1968.

[29] S. K. Chatterjea, "Fixed-point theorems," Doklady Bolgarskŏ Akademii Nauk, vol. 25, pp. 727-730, 1972.

[30] W. A. Kirk, P. S. Srinivasan, and P. Veeramani, "Fixed points for mappings satisfying cyclical contractive conditions," Fixed Point Theory, vol. 4, no. 1, pp. 79-89, 2003.

[31] M. Păcurar and I. A. Rus, "Fixed point theory for cyclic $\varphi$ contractions," Nonlinear Analysis: Theory, Methods \& Applications, vol. 72, no. 3-4, pp. 1181-1187, 2010.

[32] F. Bojor, "Fixed point theorems for Reich type contractions on metric spaces with a graph," Nonlinear Analysis: Theory, Methods and Applications, vol. 75, no. 9, pp. 3895-3901, 2012.

[33] M. A. Petric, "Some remarks concerning Ćirić-Reich-Rus operators," Creative Mathematics and Informatics, vol. 18, no. 2, pp. 188-193, 2009. 


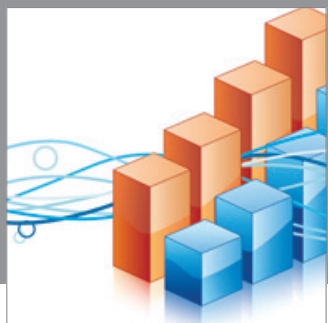

Advances in

Operations Research

mansans

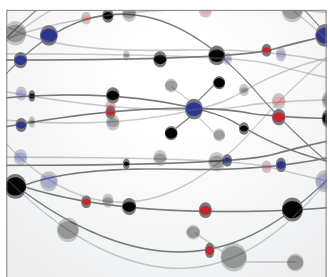

The Scientific World Journal
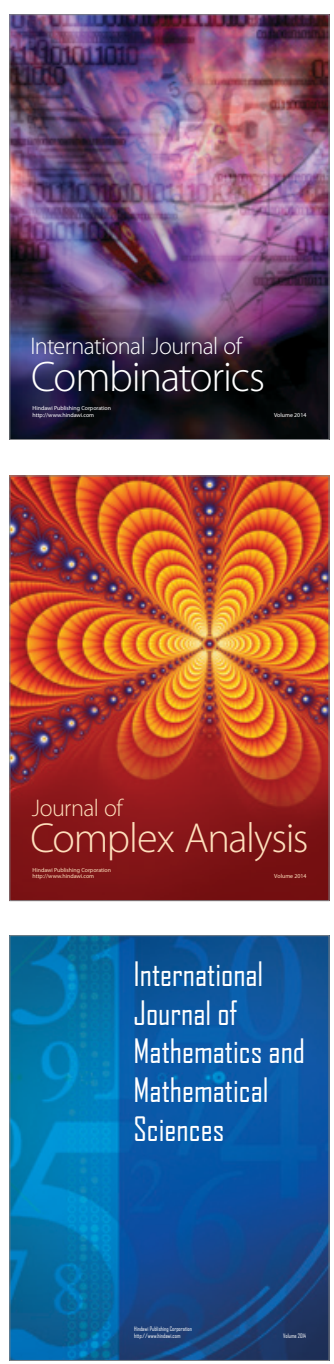
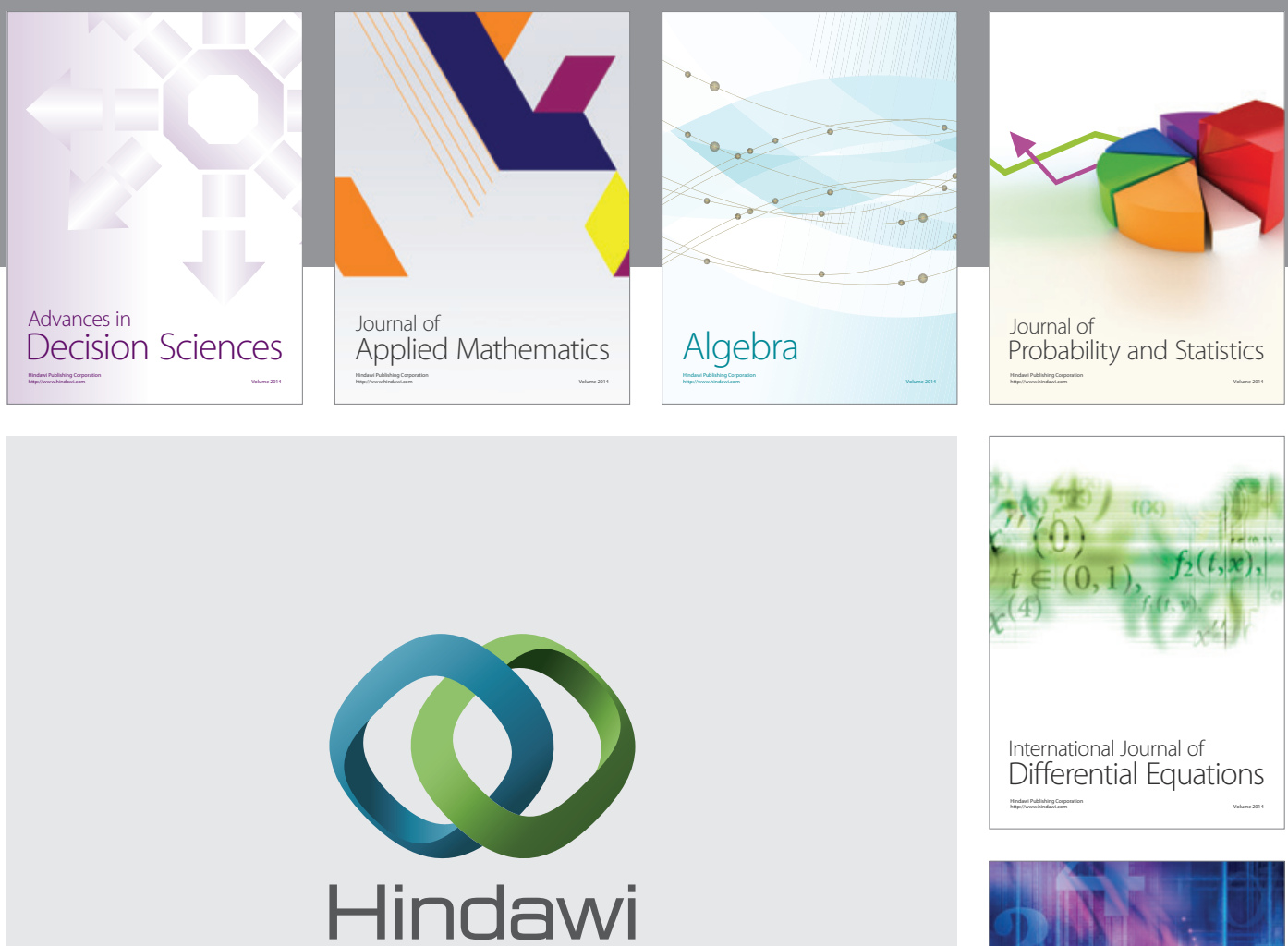

Submit your manuscripts at http://www.hindawi.com
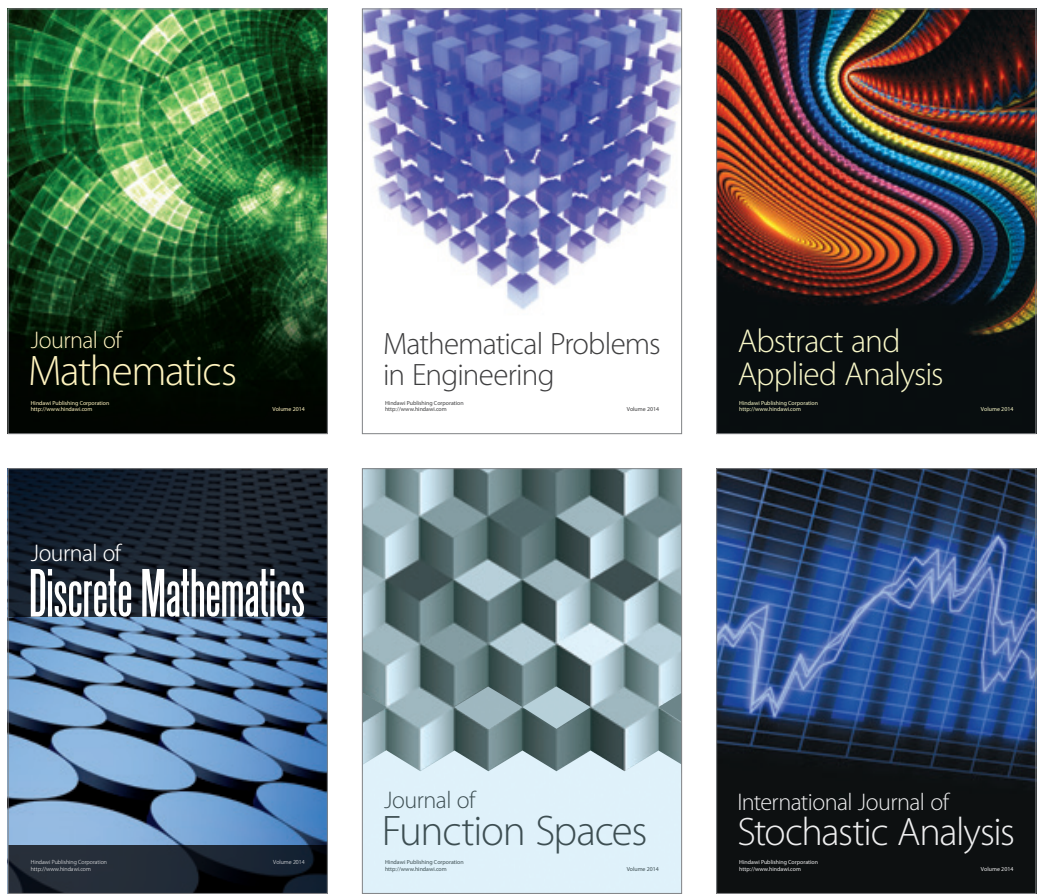

Journal of

Function Spaces

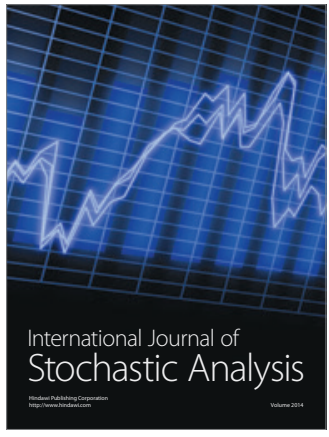

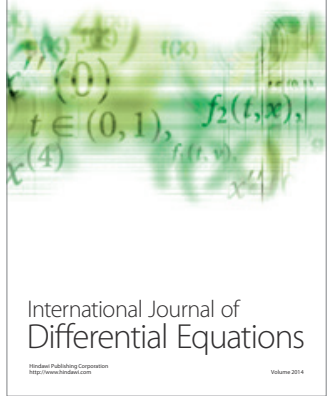
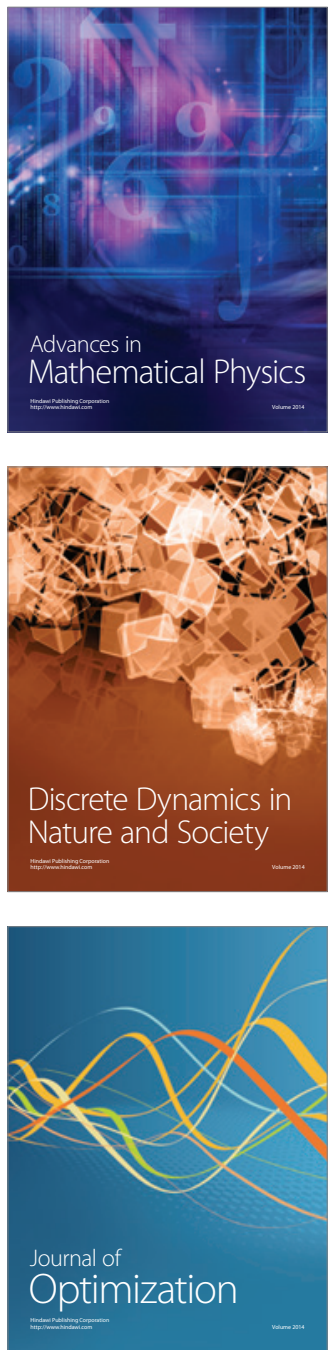